\title{
Simulating at realistic quark masses: light quark masses
}

Meinulf Göckeler ${ }^{a}$, Roger Horsley ${ }^{b}$, Yoshifumi Nakamura ${ }^{* c}$, Dirk Pleiter ${ }^{c}$, Paul E. L. Rakow $^{d}$, Gerrit Schierholz $^{c e}$, Thomas Streuer ${ }^{f}$, Hinnerk Stüben ${ }^{g}$ and James M. Zanotti $^{b}$

${ }^{a}$ Institut für Theoretische Physik, Universität Regensburg, D-93040 Regensburg, Germany

${ }^{b}$ School of Physics, University of Edinburgh, Edinburgh EH9 3JZ, UK

c John von Neumann Institute NIC / DESY Zeuthen, D-15738 Zeuthen, Germany

${ }^{d}$ Department of Mathematical Sciences, University of Liverpool, Liverpool L69 3BX, UK

${ }^{e}$ Deutsches Elektronen-Synchrotron DESY, D-22603 Hamburg, Germany

${ }^{f}$ Department of Physics and Astronomy, University of Kentucky, Lexington KY 40506, USA

${ }^{g}$ Konrad-Zuse-Zentrum für Informationstechnik Berlin, D-14195 Berlin, Germany

E-mail: meinulf.goeckeler@physik.uni-regensburg.de,

rhorsleyeph.ed.ac.uk, yoshifumi.nakamura@desy.de,

dirk.pleiter@desy.de, rakow@amtp.liv.ac.uk, gsch@mail.desy.de,

thomas.streuer@desy.de, stueben@zib.de, jzanotti@ph.ed.ac.uk

\section{QCDSF-UKQCD Collaboration}

We present new results for light quark masses. The calculations are performed using two flavours of $O(a)$ improved Wilson fermions. We have reached lattice spacings as small as $a \sim 0.07 \mathrm{fm}$ and pion masses down to $m_{\pi} \sim 340 \mathrm{MeV}$ in our simulations. This gives us significantly better control on the chiral and continuum extrapolations.

XXIVth International Symposium on Lattice Field Theory

July 23-28, 2006

Tucson, Arizona, USA

\footnotetext{
* Speaker.
} 


\section{Introduction}

The 'running' of the renormalised quark mass as the scale $M$ is changed is controlled by the $\beta$ and $\gamma$ functions in the renormalisation group equation, defined by

$$
\begin{aligned}
\beta^{S}\left(g^{S}(M)\right) & \left.\equiv \frac{\partial g^{S}(M)}{\partial \log M}\right|_{\text {bare }}, \\
\gamma_{m}^{S}\left(g^{S}(M)\right) & \left.\equiv \frac{\partial \log Z_{m}^{S}(M)}{\partial \log M}\right|_{\text {bare }},
\end{aligned}
$$

where the bare parameters are held constant. These functions are given perturbatively as power series expansions in the coupling constant,

$$
\begin{aligned}
& \beta^{S}(g)=-b_{0} g^{3}-b_{1} g^{5}-b_{2}^{S} g^{7}-b_{3}^{S} g^{9}-\ldots \\
& \gamma_{m}^{S}(g)=d_{m 0} g^{2}+d_{m 1}^{S} g^{4}+d_{m 2}^{S} g^{6}+d_{m 3}^{S} g^{8}+\ldots
\end{aligned}
$$

The first two coefficients of the $\beta$-function and first coefficient of the $\gamma_{m}$ function are scheme independent,

$$
b_{0}=\frac{1}{(4 \pi)^{2}}\left(11-\frac{2}{3} n_{f}\right), \quad b_{1}=\frac{1}{(4 \pi)^{4}}\left(102-\frac{38}{3} n_{f}\right) .
$$

and

$$
d_{m 0}=-\frac{8}{(4 \pi)^{2}}
$$

while all others depend on the scheme chosen.

We may immediately integrate eq. (1.1) to obtain

$$
\frac{M}{\Lambda^{S}}=\left[b_{0} g^{S}(M)^{2}\right]^{\frac{b_{1}}{2 b_{0}^{2}}} \exp \left[\frac{1}{2 b_{0} g^{S}(M)^{2}}\right] \exp \left\{\int_{0}^{g^{S}(M)} d \xi\left[\frac{1}{\beta^{S(\xi)}}+\frac{1}{b_{0} \xi^{3}}-\frac{b_{1}}{b_{0}^{2} \xi}\right]\right\} .
$$

The renormalisation group invariant (RGI) quark mass ${ }^{1}$ is defined from the renormalised quark mass as

$$
m_{q}^{R G I} \equiv \Delta Z_{m}^{S}(M) m^{S}(M)=\Delta Z_{m}^{S}(M) Z_{m}^{S}(M) m_{q}^{\text {bare }} \equiv Z_{m}^{R G I} m_{q}^{\text {bare }}
$$

where

$$
\left[\Delta Z_{m}^{S}(M)\right]^{-1}=\left[2 b_{0} g^{S}(M)^{2}\right]^{-\frac{d_{m 0}}{2 b_{0}}} \exp \left\{\int_{0}^{g^{S}(M)} d \xi\left[\frac{\gamma_{m}^{S}(\xi)}{\beta^{S}(\xi)}+\frac{d_{m 0}}{b_{0} \xi}\right]\right\},
$$

and so the integration constant upon integrating eq. (1.1) is given by $\Lambda^{S}$, and similarly from eq. (1.2) the integration constant is $m_{q}^{R G I} . \Lambda^{S}$ and $m_{q}^{R G I}$ are thus independent of the scale. (Note that although the functional form of $\Delta Z_{m}^{S}(M)$ is fixed, the absolute value is not; conventions vary for its definition.) Also for a scheme change $S \rightarrow S^{\prime}$ (it is now sufficient to take them at the same scale) given by

$$
g^{S^{\prime}}=G\left(g^{S}\right)=g^{S}\left(1+\frac{1}{2} t_{1}\left(g^{S}\right)^{2}+\ldots\right),
$$

\footnotetext{
${ }^{1}$ Analogous defi nitions hold for other quantities which depend on the scheme and scale chosen.
} 
$m_{q}^{R G I}$ remains invariant, while $\Lambda$ changes as $\Lambda^{S^{\prime}}=\Lambda^{S} \exp \left(t_{1} /\left(2 b_{0}\right)\right)$. Note also that analytic expressions for the integrals in eq. (1.7) or eq. (1.8) can be found for low orders, for example to two loops we have

$$
\Delta Z_{m}^{S}(M)=\left[2 b_{0}\left(g^{S}(M)\right)^{2}\right]^{\frac{d_{m 0}}{2 b_{0}}}\left[1+\frac{b_{1}}{b_{0}}\left(g^{S}(M)\right)^{2}\right]^{\frac{b_{0} d_{m 1}^{S}-b_{1} d_{m 0}}{2 b_{0} b_{1}}} .
$$

Thus we have a convenient splitting of the problem into two parts: a number, $m_{q}^{R G I}$, which involves a non-perturbative computation, and is the goal of this paper and, if desired, an evaluation of $\Delta Z_{m}^{S}$ which allows the running quark mass to be given in a renormalisation scheme $S$.

\section{Simulation}

We have estimated the light quark masses in the $\overline{M S}$ scheme at $2 \mathrm{GeV}$ by first using the axial Ward identity (AWI) to determine the lattice quark mass. This is renormalised using the RI' - MOM scheme [1] (for our variation on the method see [2]), converted to a RGI form as described in section 1 and after the continuum limit has been taken rewritten in the $\overline{M S}$ scheme. Further details and results are given in [3]. We perform our simulations with two flavours of non-perturbatively clover-improved dynamical Wilson fermions and Wilson glue. Using these actions, the QCDSF and UKQCD collaborations have generated gauge field configurations with the parameters given in Table 1. We also use configurations generated by the DIK collaboration which have been made available through the ILDG. This large set of lattices enables us to extrapolate to the chiral and the continuum limit.

\section{Quark masses}

As a first check we perform the chiral extrapolation for pseudoscalar mass. In Fig. 1 we plot $\left(a m_{p s}\right)^{2}$ against $a m_{q}^{A W I}$ together with the fit result. Our data shows that $\left(a m_{p s}\right)^{2}$ goes to 0 at $a m_{q}^{A W I}=0$. This means that even at our lightest quark mass the data is not seriously effected by either an Aoki phase or weak 1st order phase transition.

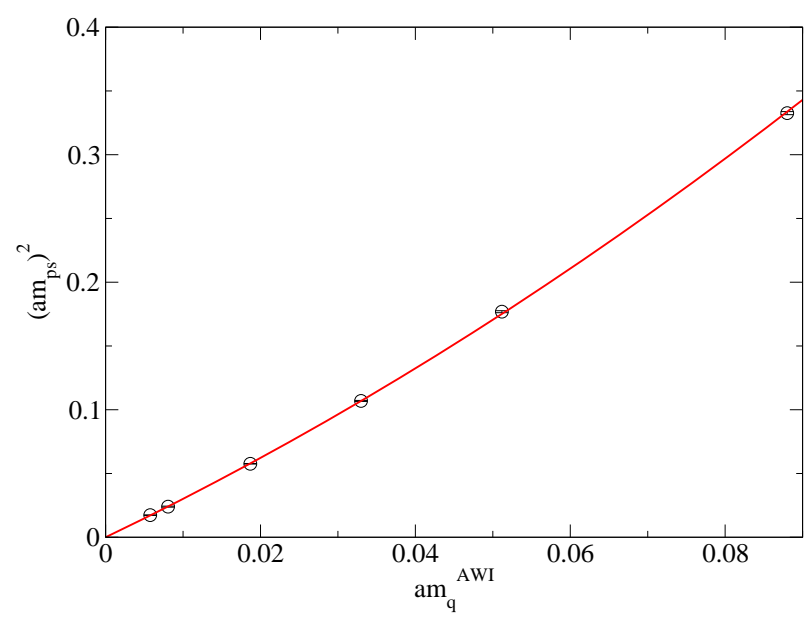

Figure 1: $\left(a m_{p s}\right)^{2}$ versus $a m_{q}^{A W I}$ for $\beta=5.29$. 


\begin{tabular}{ccccccc}
\hline \hline$\beta$ & $\kappa$ & $N^{3} \times T$ & $m_{\pi}[\mathrm{GeV}]$ & $a[\mathrm{fm}]$ & $L[\mathrm{fm}]$ & $N_{\text {traj }}$ \\
\hline 5.20 & 0.13420 & $16^{3} \times 32$ & $1.007(2)$ & 0.115 & 1.8 & $\mathscr{O}(5000)$ \\
& 0.13500 & $16^{3} \times 32$ & $0.833(3)$ & 0.098 & 1.6 & $\mathscr{O}(8000)$ \\
& 0.13550 & $16^{3} \times 32$ & $0.619(3)$ & 0.093 & 1.5 & $\mathscr{O}(8000)$ \\
\hline 5.25 & 0.13460 & $16^{3} \times 32$ & $0.987(2)$ & 0.099 & 1.6 & $\mathscr{O}(6000)$ \\
& 0.13520 & $16^{3} \times 32$ & $0.829(3)$ & 0.091 & 1.5 & $\mathscr{O}(8000)$ \\
& 0.13575 & $24^{3} \times 48$ & $0.597(1)$ & 0.084 & 2.0 & $\mathscr{O}(6000)$ \\
\hline 5.29 & 0.13400 & $16^{3} \times 32$ & $1.173(2)$ & 0.097 & 1.6 & $\mathscr{O}(4000)$ \\
& 0.13500 & $16^{3} \times 32$ & $0.929(2)$ & 0.089 & 1.4 & $\mathscr{O}(5600)$ \\
& 0.13550 & $24^{3} \times 48$ & $0.769(2)$ & 0.084 & 2.0 & $\mathscr{O}(2000)$ \\
& 0.13590 & $24^{3} \times 48$ & $0.591(2)$ & 0.080 & 1.9 & $\mathscr{O}(5000)$ \\
& 0.13620 & $24^{3} \times 48$ & $0.395(3)$ & 0.077 & 1.9 & $\mathscr{O}(3000)$ \\
& 0.13632 & $32^{3} \times 64$ & $0.337(3)$ & 0.077 & 2.5 & $\mathscr{O}(1000)$ \\
\hline 5.40 & 0.13500 & $24^{3} \times 48$ & $1.037(1)$ & 0.077 & 1.8 & $\mathscr{O}(4000)$ \\
& 0.13560 & $24^{3} \times 48$ & $0.842(2)$ & 0.073 & 1.8 & $\mathscr{O}(3000)$ \\
& 0.13610 & $24^{3} \times 48$ & $0.626(2)$ & 0.070 & 1.7 & $\mathscr{O}(4000)$ \\
& 0.13640 & $24^{3} \times 48$ & $0.432(3)$ & 0.068 & 1.6 & $\mathscr{O}(3000)$ \\
\hline \hline
\end{tabular}

Table 1: Overview of our lattice parameters. For the translation into physical units the Sommer parameter[4] with $r_{0}=0.467 \mathrm{fm}$ (see [5] and [6]) has been used.

We use the next to leading order (NLO) chiral perturbation theory $(\chi \mathrm{PT})$ to estimate the quark masses,

$$
\begin{aligned}
r_{0} m_{s}^{R G I}= & c_{a}^{R G I}\left[\left(r_{0} m_{K^{+}}\right)^{2}+\left(r_{0} m_{K^{0}}\right)^{2}-\left(r_{0} m_{\pi^{+}}\right)^{2}\right] \\
& +\left(c_{b}^{R G I}-c_{d}^{R G I}\right)\left[\left(r_{0} m_{K^{+}}\right)^{2}+\left(r_{0} m_{K^{0}}\right)^{2}\right]\left(r_{0} m_{\pi^{+}}\right)^{2} \\
& +\frac{1}{2}\left(c_{c}^{R G I}+c_{d}^{R G I}\right)\left[\left(r_{0} m_{K^{+}}\right)^{2}+\left(r_{0} m_{K^{0}}\right)^{2}\right]^{2} \\
& -\left(c_{b}^{R G I}+c_{c}^{R G I}\right)\left(r_{0} m_{\pi^{+}}\right)^{4} \\
& -c_{d}^{R G I}\left[\left(r_{0} m_{K^{+}}\right)^{2}+\left(r_{0} m_{K^{0}}\right)^{2}\right]\left[\left(r_{0} m_{K^{+}}\right)^{2}+\left(r_{0} m_{K^{0}}\right)^{2}-\left(r_{0} m_{\pi^{+}}\right)^{2}\right] \\
& \times \ln \left(\left(r_{0} m_{K^{+}}\right)^{2}+\left(r_{0} m_{K^{0}}\right)^{2}-\left(r_{0} m_{\pi^{+}}\right)^{2}\right) \\
& +c_{d}^{R G I}\left(r_{0} m_{\pi^{+}}\right)^{4} \ln \left(r_{0} m_{\pi^{+}}\right)^{2} \\
r_{0} m_{u d}^{R G I}= & c_{a}^{R G I}\left(r_{0} m_{\pi^{+}}\right)^{2}+\left(c_{b}^{R G I}+c_{c}^{R G I}\right)\left(r_{0} m_{\pi^{+}}\right)^{4}-c_{d}^{R G I}\left(r_{0} m_{\pi^{+}}\right)^{4} \ln \left(r_{0} m_{\pi^{+}}\right)^{2} .
\end{aligned}
$$

The fit function to determine $c_{a}^{R G I}$ and $c_{i}^{R G I}, i=b, c, d$ is

$$
\frac{r_{0} m_{q}^{R G I}}{\left(r_{0} m_{p s}\right)^{2}}=c_{a}^{R G I}+c_{b}^{R G I}\left(r_{0} m_{p s}^{S}\right)^{2}+c_{c}^{R G I}\left(r_{0} m_{p s}\right)^{2}+c_{d}^{R G I}\left(\left(r_{0} m_{p s}^{S}\right)^{2}-2\left(r_{0} m_{p s}\right)^{2}\right) \ln \left(r_{0} m_{p s}\right)^{2},
$$

where $m_{p s}, m_{p s}^{S}$ are the valence and sea pseudoscalar masses respectively (both using mass degenerate quarks, since we found the relevant quantities $a m_{p s}$ and $a m_{q}$ to differ by $\lesssim 1 \%$ between the degenerate quarks case and the non-degenerate quarks case). The first term is the leading order, LO, result in $\chi$ PT while the remaining terms come from the next non-leading order, NLO, in $\chi \mathrm{PT}$. 
We note that to NLO, we can determine $c_{a}^{R G I}$ and $c_{i}^{R G I}, i=b, c, d$ using mass degenerate quarks and then simply substitute them in eqs. $(3.1,3.2)$.

To reduce the total error on the result, it proved advantageous to use eq. (3.1) to eliminate $c_{a}^{R G I}$ from eq. (3.3) in terms of

$$
c_{a^{\prime}}^{R G I} \equiv \frac{r_{0} m_{s}^{R G I}}{\left(r_{0} m_{K^{+}}\right)^{2}+\left(r_{0} m_{K^{0}}\right)^{2}-\left(r_{0} m_{\pi^{+}}\right)^{2}} .
$$

This results in a modified fit function of the form

$$
\begin{aligned}
\frac{r_{0} m_{q}^{R G I}}{\left(r_{0} m_{p s}\right)^{2}}=c_{a^{\prime}}^{R G I} & +c_{b}^{R G I}\left[\left(r_{0} m_{p s}^{S}\right)^{2}-d_{b}\right]+c_{c}^{R G I}\left[\left(r_{0} m_{p s}\right)^{2}-d_{c}\right] \\
& +c_{d}^{R G I}\left[\left(\left(r_{0} m_{p s}^{S}\right)^{2}-2\left(r_{0} m_{p s}\right)^{2}\right) \ln \left(r_{0} m_{p s}\right)^{2}-d_{d}\right],
\end{aligned}
$$

where $d_{i}(i=b, c, d)$ can be read-off from eq. (3.1) and have the effect of shifting the various terms in the fit function by a constant.

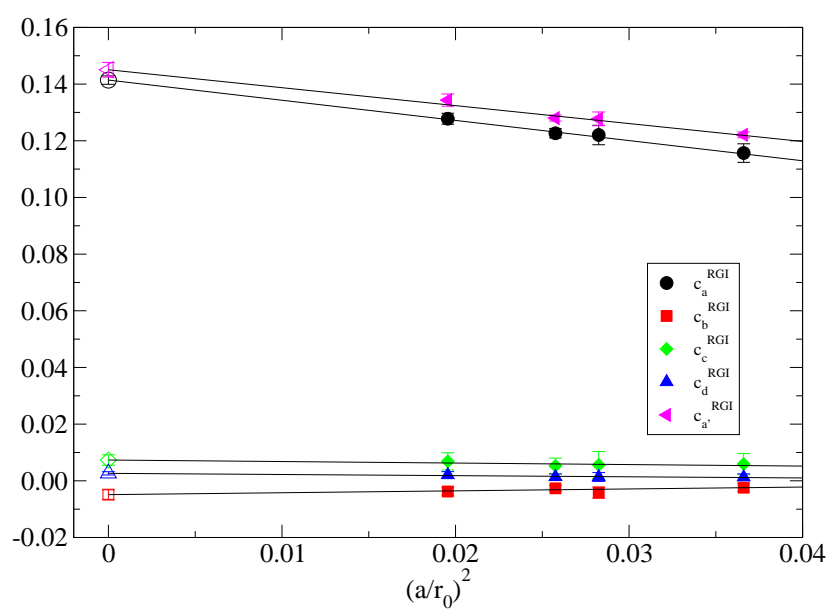

Figure 2: $c_{a}^{R G I}, c_{i}^{R G I}(i=b, c, d)$ and $c_{a^{\prime}}^{R G I}$ versus $\left(a / r_{0}\right)^{2}$. The open symbols represent the values of $c_{a}^{R G I}, c_{i}^{R G I}$ $(i=b, c, d)$ and $c_{a^{\prime}}^{R G I}$ in the continuum limit.

In Fig. 2 we plot $c_{a}^{R G I}, c_{i}^{R G I}(i=b, c, d)$ and $c_{a^{\prime}}^{R G I}$ against $\left(a / r_{0}\right)^{2}$. The coefficients of NLO are small compared to the LO coefficient. Finally, we find

$$
m_{s}^{\overline{M S}}(2 \mathrm{GeV})=\left\{\begin{array}{l}
121(2)(3)(6) \mathrm{MeV} \text { for } r_{0}=0.5 \mathrm{fm} \\
115(2)(3)(6) \mathrm{MeV} \text { for } r_{0}=0.467 \mathrm{fm}
\end{array}\right.
$$

where the first error is statistical and the second is systematic $\approx 3 \mathrm{MeV}$. We have determined it from the effect on $c_{i}^{R G I}$ by changing the fit interval $\left(r_{0} m_{p s}\right)^{2} \lesssim 5$ to $\left(r_{0} m_{p s}\right)^{2} \lesssim 4$ or 6 or $\infty$, i.e. include all the data. Furthermore the additional third (systematic) error is due to the uncertainty with which value to identify $r_{0}$.

For the light quark mass, we find that corrections from LO to NLO $\chi$ PT are negligibly small. We shall just quote the LO result of

$$
m_{u d}^{\overline{M S}}(2 \mathrm{GeV})=\left\{\begin{array}{l}
4.57(05)(07)(23) \mathrm{MeV} \text { for } r_{0}=0.5 \mathrm{fm} \\
4.34(05)(07)(23) \mathrm{MeV} \text { for } r_{0}=0.467 \mathrm{fm}
\end{array}\right.
$$




\begin{tabular}{lll} 
& \multicolumn{1}{c}{ previous } & \multicolumn{1}{c}{ new } \\
\hline$m_{s}^{\overline{M S}}(2 \mathrm{GeV})$ & $111(6)(4)(6) \mathrm{MeV}$ & $115(2)(3)(6) \mathrm{MeV}$ \\
$m_{u d}^{\overline{M S}}(2 \mathrm{GeV})$ & $4.08(23)(19)(23) \mathrm{MeV}$ & $4.34(05)(07)(23) \mathrm{MeV}$ \\
$m_{s}^{M S}(2 \mathrm{GeV}) / m_{u d}^{\overline{M S}}(2 \mathrm{GeV})$ & $27.2(3.2)$ & $26.6(1.8)$ \\
\hline
\end{tabular}

Table 2: Comparison of the updated and previous values of $m_{s}^{\overline{M S}}(2 \mathrm{GeV}), m_{u d}^{\overline{M S}}(2 \mathrm{GeV})$ and $m_{s}^{\overline{M S}}(2 \mathrm{GeV}) / m_{u d}^{\overline{M S}}(2 \mathrm{GeV})$.

where again the second and third errors are systematic. Finally, we see that the ratio

$$
\frac{m_{s}^{\overline{M S}}(2 \mathrm{GeV})}{m_{u d}^{\overline{M S}}(2 \mathrm{GeV})}=26.6(1.8) \text {. }
$$

\section{Conclusion}

We have updated our estimate for the light quark masses using results at smaller lattice spacing and smaller quark masses data. In Table 2, we compare the updated and previously published values [3]. Our results are in rough agreement with other group's results. In order to improve the precision and accuracy of analysis, simulations of smaller quark masses and lattice spacing and $2+1$ flavours are needed.

\section{Acknowledgments}

The numerical calculations have been performed on the Hitachi SR8000 at LRZ (Munich), on the Cray T3E at EPCC (Edinburgh), on the Cray T3E at NIC (Jülich) and ZIB (Berlin), as well as on the APEmille and apeNEXT at DESY (Zeuthen), while simulations at the smallest three pion masses have been performed on the BlueGeneL at NIC/Jülich, EPCC at Edinburgh and KEK at Tsukuba by the Kanazawa group as part of the DIK research programme. We thank all institutions. This work has been supported in part by the EU Integrated Infrastructure Initiative Hadron Physics (I3HP) under contract RII3-CT-2004-506078 by the DFG under contract FOR 465 (Forschergruppe Gitter-Hadronen-Phänomenologie). We would also like to thank A. Irving for providing updated results for $r_{0} / a$ prior to publication.

\section{References}

[1] G. Martinelli et al., Nucl. Phys. B445 (1995) 81, [hep-lat/9411010].

[2] M. Göckeler et al., Nucl. Phys. B544 (1999) 699, [hep-lat/9807044].

[3] M. Göckeler et al., Phys. Rev. D73, 054508 (2006), [hep-lat/0601004].

[4] R. Sommer, Nucl. Phys. B411 (1994) 839, [hep-lat/9310022].

[5] A. Ali Khan et al., hep-lat/0603028.

[6] C. Aubin et al., Phys. Rev. D70, 094505 (2004), [hep-lat/0402030]. 